INVESTIGACIONES

\title{
PERFIL DEL MENTOR BASADO EN COMPETENCIAS*
}

\author{
Mentor's profile based on competencies
}

\section{Gloria Inostroza de Celis, Enriqueta Jara, Tania Tagle}

Universidad Católica de Temuco

gloriain@uct.cl, 45+205644; ejara@uct.cl,45+205497; ttagle@uct.cl, 45+205635

\section{Resumen}

El propósito de este estudio es construir un perfil del mentor basado en competencias. La muestra estuvo constituida por once docentes que participaron de una experiencia de formación de mentores propuesta por el Ministerio de Educación de Chile y desarrollada por una universidad nacional. La información fue recogida a través de un cuestionario, entrevistas y grupo focal. Los resultados conllevan a definir un conjunto de competencias asociadas al perfil profesional de un mentor que se desempeñe en nuestro contexto educacional.

Palabras clave: formación de mentores, inducción profesional, perfil por competencias.

\begin{abstract}
The purpose of this study is to construct a mentor's profile based on competencies. The sample was formed by eleven teachers who participated in an experience of training mentors proposed by the Ministry of Education and developed by a national university. These teachers completed a questionnaire and participated in a focus group. Also some novice teachers who worked with mentors were asked to participate through an interview. The results lead to identify the competencies that would be associated to a professional profile of a mentor who works in our educational context.
\end{abstract}

Key words: mentors training, professional induction, competency based profile.

* El estudio fue desarrollado en el contexto de un Proyecto financiado por el Ministerio de Educación de Chile. 


\section{INTRODUCCION}

En la universidad se ha convivido durante décadas con la idea que el proceso de inserción profesional de los profesores principiantes era algo que dependía de la voluntad individual más que de decisiones de tipo estratégico e institucional. Esta idea, al parecer, no considera la cantidad de situaciones problemáticas que tiene que enfrentar un docente que se está iniciando en la profesión (en adelante profesor principiante) las que han sido documentadas, entre otros especialistas, por Cornejo (1999, 2001) y Marcelo (2002, 2006). Relativo a este punto, el Ministerio de Educación chileno (2006: 4) plantea "los estudios que describen la trayectoria profesional de los docentes señalan claramente que los primeros cinco años son de aprendizaje hasta que se logra seguridad y competencia docente y que, en este contexto, el primer año es decisivo, pues es el período en el que muchos docentes jóvenes deciden si abandonan la docencia o permanecen en ella”.

En este marco, surge con fuerza en nuestro contexto la necesidad de un diseño, desarrollo y evaluación de iniciativas que conlleven la implementación de prácticas de mentoría. Estas prácticas se caracterizan por establecer relaciones colaborativas entre profesor mentor y profesor principiante focalizadas en el aprendizaje y orientadas al desarrollo profesional (Lipton y Wellman, 2003). Al respecto, Marcelo (2002) plantea que un programa de mentoría e inducción demanda de un período de socialización profesional que permite al nuevo profesor la interiorización de los principios y valores de la denominada cultura de la profesión.

Teniendo en cuenta la información previa, una institución de educación superior chilena en convenio con el Ministerio de Educación desarrolló un proyecto denominado Un Estudio para el Diseño y Pilotaje de una Propuesta de Formación de Mentores de Profesores Principiantes. El proyecto tenía como objetivo esencial diseñar y validar, en un marco reflexivo constructivista, una propuesta de formación de mentores que los capacite para apoyar profesional y pedagógicamente a profesores y profesoras principiantes en su primera fase del ejercicio de la docencia. El diseño de un programa de estas características fue, sin duda, una tarea desafiante, ya que conllevó la implementación de acciones particulares dentro y fuera del aula focalizadas en mejorar el desempeño de los profesores principiantes y en apoyar su inserción constructiva a la comunidad y al sistema escolar. En este marco, una de las primeras preguntas que surgieron en el diseño de la propuesta de formación fue ¿qué perfil debe tener un profesor mentor, en el contexto chileno, para desarrollar, efectiva y eficazmente, las acciones indicadas anteriormente?

\section{MARCO TEORICO}

El proyecto desarrollado propone formar un profesor mentor estratégico, que se constituya en un profesional con habilidades regulativas para planificar, orientar y evaluar los propios procesos cognitivos relacionados con su actuación mentora (Monereo, 1998).

Para dar curso a lo anterior, el proceso formativo debía estar basado en ciertos principios u orientaciones asociados al desarrollo del aprendizaje y de la competencia profesional. Al respecto, las decisiones sobre el proceso de diseño, implementación y evaluación de la propuesta estuvieron basadas en el enfoque reflexivo (ver Dewey, 1933; Schön, 1983, 1987) y en la propuesta constructivista (ver Coll, 1990). El enfoque reflexivo 
plantea que la competencia profesional de los docentes se desarrolla a través del proceso de reflexión sobre el conocimiento recibido (derivado de hechos y teorías relacionadas a una determinada actividad profesional) y el conocimiento experiencial (derivado de las experiencias de enseñanza) a la luz de la acción profesional (Wallace, 2001). Por su parte, la propuesta constructivista plantea que las personas aprenden en la medida que se producen relaciones significativas entre los conocimientos previos que poseen y la nueva información que se les presenta (Malderez y Bodóczky, 2002). Relativo al punto previo, Williams y Burden (2001) plantean que en el constructivismo no existiría una forma apropiada de enseñar, ya que la preocupación más relevante de esta práctica social sería desarrollar el aprendizaje.

Como se ha sugerido con antelación, el proceso de formación de los docentes para transformarse en mentores es una tarea desafiante, pues requieren construir conocimientos y desarrollar habilidades y actitudes que van más allá de aquellas necesarias para ejercer el rol profesional. Ellos necesitan apoyar a los profesores principiantes a aprender de sus propias experiencias, lo que significa:

- reconocer y tratar de comprender la complejidad involucrada en la práctica profesional,

- conocer cómo la investigación sobre la enseñanza y el aprendizaje pueden iluminar la práctica,

- conocer cómo investigar la práctica y la teoría que subyace a la misma,

- comprender cómo la práctica individual se relaciona con aspectos morales de la práctica profesional y reconocer las implicancias de esto,

- ser capaces de ayudar a develar las creencias, presupuestos y valores que subyacen o están a la base de la práctica de los profesores principiantes,

- ser capaces de ayudar a los profesores principiantes a encontrar o identificar nuevos enfoques para orientar la práctica,

- ser capaces de comprometerse en discusiones con los profesores principiantes sobre los propósitos de la educación.

(Tomado de Turner y Bash, 1999)

En este contexto, Malderez y Wedell (2007) indican que un mentor tiene que desempeñar diferentes roles cuando desarrolla su función profesional. Estos autores identifican estos roles como: acculturator, model, support, sponsor y educator. Así, cuando un mentor actúa como acculturator ayuda a los profesores principiantes a ser conscientes y a comprender la cultura de la institución educacional en la cual ellos desarrollan su actividad profesional. Por otro lado, actúa como model cuando modela actitudes positivas que influyen las formas en que los docentes se aproximan al aprendizaje, se comprometen con su propio desarrollo profesional y construyen relaciones profesionales con colegas, padres, etcétera. En este mismo sentido, actúa como support cuando construye una relación de confianza y cercana con los profesores principiantes mostrando compromiso con su desarrollo personal y profesional. Por otra parte, se desempeña como sponsor cuando apoya a los profesores principiantes para ser aceptados en la comunidad profesional de la institución educacional en la cual ellos están desarrollando su función. Finalmente, se desempeña como educator cuando media o genera los andamiajes necesarios para que los profesores principiantes logren construir sus aprendizajes. 
De acuerdo a lo anterior, aquellos participantes involucrados en un curso de formación de mentores deben:

- tener oportunidad de explorar las propias creencias y actitudes que subyacen a los procesos de aprendizaje, enseñanza y mentoría,

- poseer el conocimiento, habilidades y recursos para ayudar/apoyar a los profesores principiantes a planificar intervenciones pedagógicas,

- presentar habilidades para evaluar el desempeño de los docentes teniendo como referencia ciertos estándares,

- contar con habilidades para conducir conversaciones posteriores a las intervenciones pedagógicas de tal forma que los profesores principiantes lleguen a ser capaces de autoevaluarse, formulando planes de acción y permitiendo el continuo desarrollo profesional de los mismos,

- construir conocimiento y desarrollar estrategias que les permitan su propio desarrollo profesional.

(Bodóczky y Malderez, 1996)

Tomando como base estas características, el objetivo de la formación de los mentores del estudio se relaciona con el desarrollo de competencias, a través de la definición de un perfil competencial. Esta opción se sustentaría en tres aspectos:

- El primero de ellos asociado principalmente al avance de la educación hacia escenarios cada vez más diversos e inciertos en los cuales el principal desafío consiste en encontrar modos de desarrollo humano que permitan a las personas enfrentar efectivamente las brechas ocasionadas por la modernidad en un mundo cada vez más global y, en tal sentido, quienes desarrollan actividades docentes deben estar preparados para desempeñarse en estos contextos (Morín, 1999).

- En segundo lugar, la complejidad de los espacios socioculturales y laborales actuales demandan cambios fundamentalmente cualitativos y de mayor énfasis en las competencias de los docentes, para que puedan convertirse en sujetos de su aprendizaje, preparados en y para la vida, y capaces de aportar a la sociedad en que les tocará desempeñarse (Marcelo, 1999). De este modo, los profesores principiantes que se integren al sistema escolar necesitarán vivenciar procesos de mentoría que les ayuden en la movilización de saberes referidos tanto al hacer, como al saber vivir y al saber ser de su profesión (Delors, 1996). Lo anterior les permitirá poner en acción sus conocimientos/recursos personales para enfrentar la vida en general y/o para resolver las situaciones problemáticas que puedan emerger en el ejercicio profesional y en la actuación social manifestando un alto compromiso ético por lo que realizan.

- En tercer lugar, y en base a la realidad descrita, la formación del mentor requiere crear experiencias de aprendizaje para que éstos construyan competencias que les permitan movilizar, de forma integral, recursos indispensables para desarrollar de manera óptima las acciones demandadas al profesor principiante. El paradigma que sustenta una opción formativa de esta índole implica cambios profundos en la forma de concebir el desempeño, la evaluación y el desarrollo de la función mentora, otorgando claridad a los procesos que involucran la acción y distinguiendo los saberes que se pondrán en juego en la actuación profesional, disminuyendo la incertidumbre 
que puede suponer el ejercicio del rol mentor que en Chile está progresivamente emergiendo.

Frente a este marco de necesidades, es importante señalar que en consideración a las múltiples acepciones existentes en torno al término competencia, uno de los desafíos fue seleccionar y asumir aquélla que presentara mayor coherencia con los propósitos de una formación de mentores en el escenario educativo actual. Algunas de las definiciones existentes son las discutidas por los siguientes autores.

Bunk (1994: 16) sostiene que "posee competencia profesional quien dispone de los conocimientos, destrezas y aptitudes necesarios para ejercer una profesión, puede resolver los problemas profesionales de forma autónoma y flexible, está capacitado para colaborar en su entorno profesional y en la organización del trabajo". Le Boterf (1998: 23), por su parte, la reconoce como "una construcción, a partir de una combinación de recursos (conocimientos, saber hacer, cualidades o aptitudes), y recursos del ambiente (relaciones, documentos, informaciones y otros) que son movilizados para lograr un desempeño". Para Perrenoud (2004), es posible entender la competencia como el conjunto de conocimientos, destrezas y actitudes que ha de ser capaz de movilizar una persona, de forma integrada, para actuar eficazmente ante las demandas de un determinado contexto.

En consideración a lo anterior y en el marco del proyecto desarrollado, la competencia es entendida como 'un saber actuar movilizando recursos propios (asociados al saber, saber hacer y saber ser), y del entorno para resolver problemas o satisfacer necesidades reales emanadas del contexto profesional'. En tal sentido, las competencias tienen un nivel de complejidad superior al de los principales atributos, pues integran un repertorio más amplio de destrezas.

En síntesis, el estudio reconoce las siguientes características de la competencia en esta opción formativa de mentores:

- Se ejercita en un contexto real: toda competencia se desarrolla en un contexto determinado con todas las variables que éste involucra.

- Se ubica en un continuo que va de lo sencillo a lo complejo.

- Pertenece al orden del saber movilizar en el quehacer profesional: si bien es cierto se requiere disponer de los distintos tipos de saberes como recursos para fundamentar la competencia se requiere de contextos reales para identificar la realización de la misma, es así como el contexto real exige que el individuo competente sepa interpretar y analizar la situación, detectar los recursos disponibles y ejecutar una acción integrando efectivamente y en forma pertinente todos los elementos.

- Como saber-actuar es inherente a una práctica intencional: la competencia como saber actuar permite el logro de objetivos considerados como deseables de lograr.

- Es un saber-actuar logrado, eficaz, ágil e inmediato que se manifiesta en forma recurrente: la competencia se manifiesta por una ejecución eficaz y expedita realizándose de manera recurrente en diversas situaciones indicando que el saber-actuar está estabilizado.

- Constituye un proyecto, una finalidad sin fin: la competencia, como se mencionó va de lo sencillo a lo complejo, pero la complejidad máxima no constituye un fin, en otras palabras, no se puede manifestar que alguien haya logrado plenamente una competencia ya que siempre se manifiestan indicios de superación de la misma. 
- La persona competente se sustenta en un esquema de recursos: el profesional competente recurre a distintos recursos personales y del entorno movilizándolos adecuadamente en el contexto de la acción, los recursos personales, a su vez, están asociados con el saber, saber hacer y con las actitudes que demanda un determinado rol profesional.

(Martinet, Raymond y Gauthier, 2004)

En este marco, la configuración del perfil del mentor se constituye por la descripción del conjunto de atributos que éste evidencia en el ejercicio de su rol para desempeñarse con un grado de eficiencia razonable en el cumplimiento de acciones propias del proceso de acompañamiento del profesor principiante.

Se produce de esta forma una estrecha relación entre el perfil y las competencias, puesto que son éstas las que se definen y organizan orientando las decisiones respecto de la ruta formativa a seguir para que el profesional en formación sea reconocido por el entorno laboral y social en función de los impactos que producen los procesos de apoyo que brinda al profesor principiante para su exitosa inserción profesional.

Relativo a este último punto, es importante mencionar que se parte del supuesto que para que el profesor mentor logre control sobre su propio proceso de aprendizaje requiere de una formación de competencias estratégicas que le posibiliten la adopción de decisiones adecuadas a partir de reflexiones y razonamientos basados en principios y en procedimientos de intervención acordes a las necesidades de los profesores principiantes.

\section{METODOLOGIA}

La opción de levantar un perfil de formación por competencias para el mentor se sustenta en el marco de una opción pedagógica de desarrollo integral, lo cual se potencia con una aproximación pedagógica que permite superar el atomicismo de los programas de formación tradicionales englobando las dimensiones del saber, saber hacer y saber ser, presentes en el desempeño del profesor mentor.

De este modo, se hizo necesario levantar un perfil que permitiera reconocer aquellos aspectos que un mentor debe considerar en su función. En tal sentido, es importante destacar que éste constituye la descripción del conjunto de atributos que el mentor evidencia en el ejercicio de su rol para desempeñarse con eficiencia y eficacia en el cumplimiento de acciones propias del proceso de acompañamiento del profesor principiante.

En este contexto, y como primera aproximación a la tarea de definición de competencias asociadas al perfil de un mentor y como marco de referencia general para orientar el proceso de diseño de la propuesta de formación, se identificaron competencias asociadas a las áreas: mediación profesional y gestión. Estas áreas representan conjuntos de competencias agrupadas en función de ámbitos sectoriales en las cuales el mentor debe desarrollar su quehacer y se explicitan de la siguiente forma:

\section{a) Mediación profesional}

El profesor mentor apoya el desarrollo profesional del profesor principiante a través del acompañamiento para la construcción de distintos tipos de saberes asociados a la cultura escolar donde éste se inserte. Para estos efectos el profesor mentor en forma colaborativa 
organiza y desarrolla procesos de andamiaje que, enfocados en situaciones de la vida real y de la experiencia del profesor principiante, le aportan instrumentos para interpretar y analizar situaciones profesionales para las cuales decide y en consecuencia actúa.

De este modo, y en el marco de una relación dinámica y recíproca, el mentor pone en marcha procesos que permiten al profesor principiante afianzar habilidades de automonitoreo de su propio desempeño profesional asumiendo los aciertos, errores y debilidades como indicadores de proceso y, por tanto, como fuentes de aprendizaje.

\section{b) Gestión}

El profesor mentor se caracteriza por ser un profesional que moviliza recursos propios y ajenos para la conformación de redes que faciliten la inserción profesional del profesor principiante a sus contextos de desempeño. Por esto último, diseña, organiza e implementa acciones con docentes, apoderados y otros actores significativos de la comunidad para en un trabajo asociado generar aprendizajes de calidad.

El levantamiento de las competencias asociadas a las áreas previamente identificadas y definidas estuvo basado esencialmente en la literatura especializada quedando el perfil originalmente establecido con las competencias que se identifican en Anexo 1. A cada una de las competencias especificadas se le asociaron ciertos saberes (conceptuales, procedimentales y actitudinales).

En este marco, es relevante mencionar que lo primero que se realizó fue la aplicación y análisis de la información recogida a través de un cuestionario que fue desarrollado por once profesores mentores que estaban participando de la experiencia. Esta aplicación se realizó al finalizar el componente inicial de formación profesional (que obedecía a la organización modular) y justo antes de comenzar el trabajo directo con el profesor principiante. Constituyéndose este proceso en uno de validación inicial de las competencias desde la perspectiva de los profesores mentores participantes lo que, a su vez, conllevaba una revisión y análisis de los referentes que sustentaban la propuesta.

Relativo a esto es importante destacar que las competencias se validaron definitivamente en la fase de encuentro y de colaboración del profesor mentor con el profesor en los contextos profesionales reales donde el primero conjugó y movilizó distintos tipos de saberes construidos en el proceso de mediación profesional que realizó para que el profesor principiante construyera aprendizajes significativos. Dentro de este marco es relevante mencionar que una vez que se hubo iniciado el trabajo de mentoría se aplicó una entrevista a los profesores principiantes y se realizó un grupo focal con los profesores mentores una vez finalizado el proceso.

En el mismo sentido, en este proceso de validación participó la Comisión de Inducción del Centro de Perfeccionamiento e Investigaciones Pedagógicas de Ministerio de Educación (CPEIP), la que junto con emitir su juicio experto desarrolló acciones de retroalimentación que aportaron al levantamiento del referencial de competencias definitivo.

\section{RESULTADOS}

Los procesos específicos de validación de las competencias se presentan a continuación. 
En relación a la información recogida a través del cuestionario, un primer aspecto a considerar es que la mayoría de las competencias originales fueron valoradas en el rango de muy importante o bastante importante. Sólo la competencia ocho que indica que el profesor mentor "asesora al profesor principiante en la implementación y evaluación de diseños pedagógicos”, presentó dos valoraciones respecto a considerarla poco importante. Para ilustrar los datos, se presenta un gráfico que representa el nivel de valoración muy importante otorgado por los mentores.

\section{Gráfico 1}

Valoración general respecto a nivel muy importante

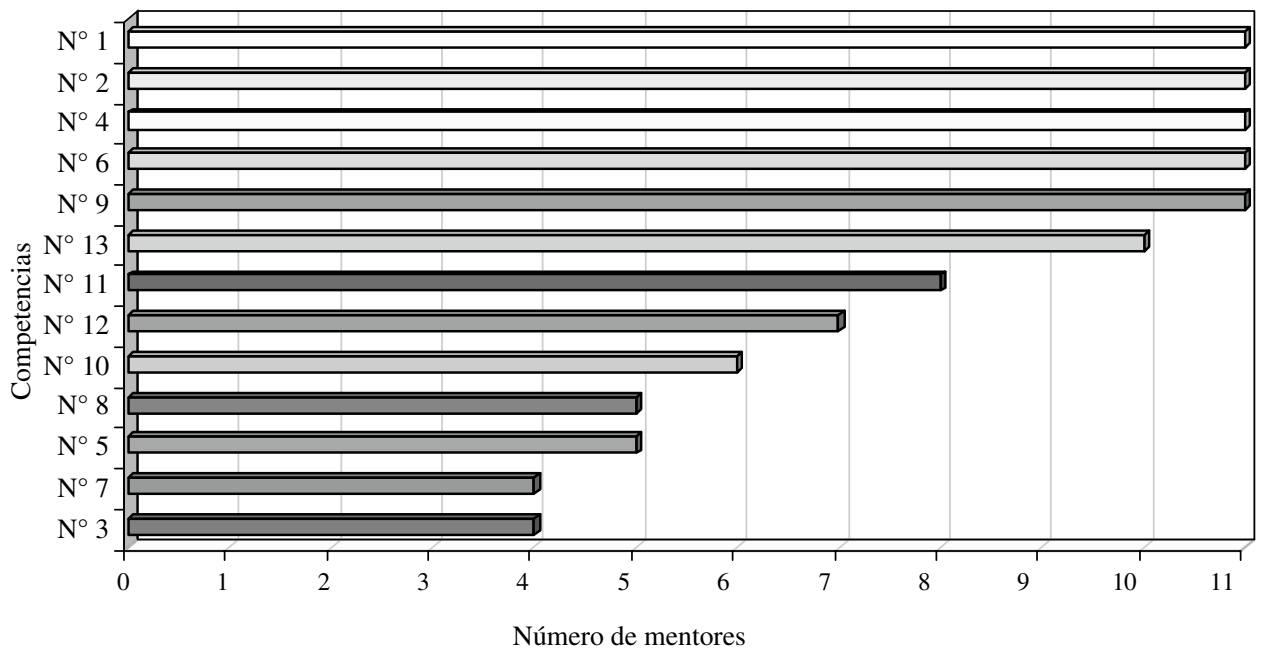

Del gráfico, se observa que las competencias que aparecen menormente consideradas como muy importantes son la número tres y siete, referidas a: "evalúa el ámbito sociocultural de desempeño del profesor principiante", y "asesora al profesor principiante en diseños pedagógicos pertinentes a los estudiantes y al contexto". Es interesante destacar que éstas se asocian a aspectos diferentes del ejercicio del rol de mentor, puesto que mientras la primera se orienta al conocimiento que éste debe tener de la realidad en la cual trabaja el principiante, a fin de tomar las decisiones pertinentes para las acciones de apoyo, la otra se refiere a aspectos de la didáctica que implementa el principiante. Sin embargo, sí se puede reconocer que las dos acogen el sentido del contexto, lo que puede resultar interesante de analizar a la luz de cómo ve el mentor los espacios de acción en su desempeño.

Otro de los aspectos evaluados a través del cuestionario fue el referido a la prioridad que cada mentor le asignaba a las competencias. Al respecto, es importante destacar que si bien puede parecer innecesaria la ordenación de las competencias según la preferencia que cada uno de los mentores le otorgaba, resulta interesante conocer cómo ellos la visualizaban en el ejercicio de su rol. 
Las competencias priorizadas se orientaron tanto al área de mediación y de gestión. En cuanto al área de mediación, se encuentran en primer lugar dos competencias "genera un ambiente facilitador para el proceso de mediación profesional del profesor principiante" y "comunica ideas en los diversos escenarios en que realiza el proceso de mediación", luego en segundo lugar se repitió la competencia uno, y en tercer lugar se identificó "genera en el profesor principiante la construcción de aprendizajes significativos". En el caso del área de 'Gestión' se reconoce en cuarto lugar la competencia referida a "promueve que el profesor principiante se constituya en agente de cambio".

Como se indicó con antelación, se continúo la validación definitiva del perfil a través de un monitoreo constante del proceso en los contextos reales de implementación del proyecto. Dadas las características del estudio se combinaron métodos, enfatizando las características del análisis cualitativo y su estrecha relación del trabajo y el entorno desde situaciones ocupacionales concretas de cada mentor con su respectivo profesor principiante.

Relativo al punto previo, en la información recopilada a través de las entrevistas, los profesores principiantes relevaron dimensiones asociadas tanto al área de mediación como a la de gestión. En este marco, se destacaron aspectos relacionados con el saber, saber hacer y ser del profesor mentor, enfatizándose que posea expertiz en su área de especialidad, que manifieste el desarrollo de habilidades comunicativas, del pensamiento y de liderazgo, y que evidencie compromiso con la profesión. Todos estos aspectos, a su vez, se asocian con el rol colaborador que se espera que este profesional desarrolle. Relacionado al punto previo, es importante mencionar que cada una de las características personales y/o profesionales que fueron relevadas por los profesores principiantes fueron a su vez consideradas en las competencias asociadas a la formulación del perfil original.

Una vez que el proceso de mediación profesional del profesor mentor estaba próximo a finalizar, se realizó grupo focal con los profesores mentores. En este marco, ellos coincidieron en indicar que la mayoría de las competencias se evidenciaban en la práctica como relevantes, destacando que se pueden observar en el ejercicio del rol profesional; sin embargo, a partir de algunos datos recopilados se pudo inferir que el tiempo destinado a la práctica de mentoría (un semestre) era insuficiente para evidenciar la competencia número doce en el desempeño profesional, la cual establece que el mentor "genera redes para el encuentro del profesor principiante con su realidad laboral y los contextos de desempeño".

Respecto a lo anterior, es importante indicar que algunas de las competencias que fueron particularmente discutidas por los mentores en el grupo focal fueron: la tres "Evalúa el ámbito sociocultural de desempeño del profesor principiante", la siete "Asesora al profesor principiante en diseños pedagógicos pertinentes a los estudiantes y al contexto" y la ocho "Asesora al profesor principiante en la implementación y evaluación de diseños pedagógicos".

Lo anterior se relaciona con los resultados asociados al cuestionario en función de la valoración dada por los profesores a las competencias en torno a la opción muy importante. En síntesis, llamó la atención que las competencias que fueron especialmente discutidas en los resultados del cuestionario fuesen nuevamente tema de discusión en el foco grupal.

Relativo al punto previo, en el grupo focal se tuvo la posibilidad de profundizar o de recoger información con respecto a la percepción de los profesores mentores sobre 
las competencias anteriormente indicadas. Acerca de la número tres, por un lado, algunos docentes consideran que es importante porque genera oportunidades para que el mentor indague, conozca y comprenda la realidad sociocultural en la que se desempeña profesionalmente el profesor principiante, pero a otros les generaba ruido porque desde su percepción en su redacción no hacía alusión al encuentro entre el profesor mentor y el profesor principiante, es decir, la competencia se vería redactada desde una lógica distinta a la naturaleza propia de las relaciones de mentoría las que indican la idea de encuentro. Relativo a las competencias número siete y ocho, es relevante destacar que ambas, a diferencia de las once restantes, parecen no ser transversales a toda acción profesional de un profesor mentor.

Por otro lado, en función del área de gestión se destaca principalmente la competencia asociada en promover que el profesor principiante sea gestor de cambio, la relevancia de esta competencia también quedó demostrada en las respuestas que dieron los docentes en el cuestionario, ya que salió la cuarta más votada entre las trece y la primera entre las del área.

En el mismo sentido, se puede destacar lo expresado por los profesores principiantes los cuales asignaron importancia a ambas áreas de competencias, pero resaltaron especialmente las competencias número uno que sugiere que el profesor mentor "genera un ambiente facilitador para el proceso de mediación profesional del profesor principiante" y la número seis que señala "guía al profesor principiante en el proceso de autoevaluación de su propio desempeño profesional'. Lo anterior se puede asociar con lo que Marcelo (2006: 16) sugiere cuando señala que "parece evidente, y así lo constatamos en los distintos estudios revisados, que los profesores mentores que van a trabajar con profesores principiantes deben poseer ciertas características personales (empatía, facilidad para la comunicación, paciencia, diplomacia, flexibilidad, sensibilidad) ...las cualidades que posea el profesor mentor van a influir en las relaciones que establezca con el profesor principiante..."

También Lipton y Wellman (2003) indican que la seguridad emocional es necesaria para producir la complejidad cognitiva. Ellos indican que poner atención en el ambiente intelectual, físico y emocional en la relación de mentoría acelera el crecimiento del novicio al experto. Añaden además que los mentores deben construir un espacio seguro, donde la comunicación verbal y no verbal indique atención completa, altas expectativas, y un cuidadoso balance entre desafío y apoyo. Estos componentes permitirían al mentor y profesor principiante compartir preguntas, información y preocupaciones en interacciones productivas, de apoyo y confidenciales. En el mismo sentido se plantea Sandlin (2007) al reconocer que se dedica mucho tiempo con el mentor sobre cómo trabajar con el principiante y no decirle cómo lo está haciendo.

De acuerdo a lo señalado por los profesores principiantes, el apoyo del mentor puede ser determinante para un cambio positivo de la práctica, pues al ayudarles a analizar y comprender sus propios procesos de inserción laboral pueden avanzar en su desarrollo profesional.

Por otro lado, es relevante mencionar que se pudo identificar una valoración importante asignada a algunas competencias que no fueron incluidas explícitamente, éstas son: compromiso ético y gestión del tiempo. Respecto a la primera, resulta importante mencionar que en el perfil originalmente propuesto se consideró transversal a todas las competencias que debía desarrollar un mentor en su contexto profesional; sin embargo, 
al omitir su explicitación su presencia se hizo difusa. La relevancia de la dimensión ética en el proceso de formación de mentores es relevada por varios especialistas, entre ellos Orland-Barak (2007). Esta autora sugiere que en muchas ocasiones los mentores se identifican con su propio código ético como docentes y el mismo puede diferir o pueden estar en tensión con los códigos del profesor principiante o con los del contexto en que se desempeña este último por lo que señala que es una dimensión importante a trabajar.

Relativo a la competencia asociada a la gestión del tiempo, Marcelo (2006) plantea que los programas de inserción que han demostrado ser eficaces involucran reducción de tiempo o de carga docente para profesores principiantes y mentores. En este mismo marco, Malderez y Wedell (2007) sugieren que los mentores requieren tiempo para escuchar a los profesores principiantes hablar sobre su práctica pedagógica, para guiarlos en el proceso de revisión de la misma y para apoyarlos en la toma de decisiones informadas de lo que harán en el futuro (planificar la acción profesional próxima). Al parecer, entonces, se requiere saber gestionar apropiadamente el tiempo disponible ya que parece ser una competencia genérica importante en toda profesión (Villa y Poblete, 2007).

\section{CONCLUSIONES}

Los resultados de este estudio permitieron identificar las competencias de un mentor, de tal modo que sirvan de referencia para guiar nuevas acciones de perfeccionamiento de ellos así como la actualización permanente de las herramientas de formación, en coherencia a los cambios socioeducativos y culturales de desempeño docente que se requieran.

Porque la complejidad de la realidad educativa actual destaca la necesidad de orientar el proceso educativo hacia el aprendizaje, y en ella la transición que realiza el profesor principiante constituye un proceso que requiere andamiajes que permitan, a quien se inicia en la profesión docente, movilizar los saberes construidos en el desarrollo de su identidad profesional.

En este marco, y bajo las características ya descritas, realizado el proceso de validación del perfil y de sus componentes fundamentales (ver Anexo 2), las competencias fueron identificadas con los respectivos saberes (ser, hacer, saber) esenciales que promueven su desarrollo. En relación al ser, éste se asocia a la disposición personal afectiva y/o cognitiva que moviliza un comportamiento determinado y que está a la base del saber y saber hacer. El hacer se refiere a las habilidades o procedimientos complejos caracterizados por haber alcanzado un nivel de dominio o pericia en el manejo de estrategias o técnicas propias del ámbito profesional. Por su parte, el saber se relaciona a los conceptos y principios y leyes cuyas conceptualizaciones son indispensables para explicar, fundamentar y proyectar la acción.

Finalmente, lo anterior implica desafíos tanto para las universidades como para los responsables de los espacios de acción docente. En el primer caso, la formación inicial debe integrar en su itinerario formativo espacios de aprendizaje asociados a la colaboración y la inserción profesional en contextos de desempeño diversos. En relación al Ministerio de Educación y sostenedores de la educación, les demanda potenciar un capital humano avanzado que pueda convertirse en un apoyo para el nuevo profesor a fin de que éste disponga de acompañamiento para controlar, organizar, modificar y monitorear sus propios procesos de transición laboral. 


\section{BIBLIOGRAFIA}

Bodóczky, C. \& A. Malderez (1996). Out into schools. En Medgyes, P. y Malderez, A (eds), Changing perspectives in teacher education. Great Britain: Heinemann.

Bunk, G. P. (1994). La transmisión de las competencias en la formación y perfeccionamiento profesionales en la RFA, Revista CEDEFOP, $\mathrm{N}^{\circ} 1$.

Coll, C. (1990). Aprendizaje escolar y construcción del conocimiento. Barcelona: Paidós.

Cornejo, J. (1999). Profesores que se inician en la docencia: algunas reflexiones al respecto desde América Latina, Revista Iberoamericana de Educación, 19, pp. 1-35.

Cornejo, J. (2001). Inserción e iniciación docente de profesores principiantes: estudio de seguimiento en un programa colaborativo entre la universidad y el medio escolar, Boletín de Investigación Educacional, $\mathrm{N}^{\circ} 16$, pp. 77-93.

Delors (1996). La educación encierra un tesoro. Informe de la Comisión de la UNESCO para la Educación en el Siglo XXI. Madrid: Santillana.

Dewey, J. (1933). How we think. London: D.C. Health Co.

Le Boterf, G. (1998). La ingeniería de las competencias, D'organisation, $\mathrm{N}^{\circ} 6$, p. 23.

Lipton, L. \& B. Wellman (2005). Mentoring matters: a practical guide to learning-focused relationships. USA: Mira Via.

Malderez, A. \& C. Bodóczky (2002). Mentor courses: a resource book for trainer-trainers. Cambridge: Cambridge University Press.

Malderez, A. \& M. Wedell (2007). Teaching teachers: processes and practices. London: Continuum.

Marcelo, C. (1999). Formación del profesorado para el cambio educativo. Barcelona: Ed. EUB.

Marcelo, C. (2002). Los profesores como trabajadores del conocimiento. Certidumbre y desafíos para una formación a lo largo de la vida, Educar, $\mathrm{N}^{\mathrm{o}} 30$, pp. 27-65.

Marcelo, C. (2006). Políticas de inserción a la docencia: de eslabón perdido a puente para el desarrollo profesional docente. Informe elaborado para PREAL (Programa para la Reforma Educativa en América Latina), Grupo de Trabajo sobre Profesionalización Docente, y presentado el 23 de noviembre en el Seminario: Políticas para integrar a los nuevos profesores en la profesión docente.

Martinet, M., D. Raymond y C. Gauthier (2004). Formación de docentes: orientaciones, competencias profesionales. Québec: Ministerio de Educación.

MINEDUC (2006). Términos de referencia para una propuesta de formación de mentores. Santiago: MINEDUC.

Monereo, C. (1998). Estrategias de enseñanza y aprendizaje. Barcelona: Graó.

Morín, E. (1999). Los siete saberes necesarios a la educación del futuro. París: UNESCO.

Orland-Barak, L. (2007). Aprender a ser mentor: de la teoría a la práctica. Seminario Interno sobre Formación de Mentores, Universidad Católica de Temuco (paper).

Perrenoud, P. (2004). Diez nuevas competencias para enseñar. Barcelona: Graó.

Sandlin, R. (2007). El sistema de Mentoría en California. Seminario sobre Formación de Mentores, MINEDUC (paper).

Schön, D. A. (1983). The reflective practitioner: how professionals think in action. London: Temple Smith.

Schön, D. A. (1987). Educating the reflective practitioner: toward a new design for teaching and learning in the professions. San Francisco: Jossey-Bass Publishers.

Turner, M. \& L. Bash (1999). Sharing expertise in teacher education. London: Cassell.

Villa, A. y Poblete (2007). Aprendizaje basado en competencias. Una propuesta para la evaluación de competencias genéricas. Bilbao: Mensajero.

Wallace, M. (2001). Training foreign language teachers: a reflective approach. Cambridge: Cambridge University Press.

Williams, M. \& Burden, R. (2001). Psychology for language teachers: a social constructivist approach. Cambridge: Cambridge University Press. 


\section{ANEXO 1}

Areas y competencias iniciales

\section{Mediación profesional}

1. Genera un ambiente facilitador para el proceso de mediación profesional del profesor principiante.

2. Comunica ideas en los diversos escenarios en que realiza el proceso de mediación

3. Evalúa el ámbito sociocultural de desempeño del profesor principiante.

4. Genera en el profesor principiante la construcción de aprendizajes significativos.

5. Guía al profesor principiante en la evaluación de sus estudiantes y de su contexto de desempeño.

6. Guía al profesor principiante en el proceso de autoevaluación de su propio desempeño profesional

7. Asesora al profesor principiante en diseños pedagógicos pertinentes a los estudiantes y al contexto.

8. Asesora al profesor principiante en la implementación y evaluación de diseños pedagógicos.

9. Distingue acciones de apoyo crítico para la autogestión del desarrollo profesional del profesor principiante.

10. Evalúa el impacto de los procesos de mediación profesional implementados.

\section{Gestión}

11. Promueve que el profesor principiante se constituya en agente de cambio.

12. Genera redes para el encuentro del profesor principiante con su realidad laboral y los contextos de desempeño.

13. Gestiona su desempeño profesional desde una concepción de asesoría y apoyo al profesor principiante.

\section{ANEXO 2}

Referencial de competencias definitivo

1. Manifiesta compromiso ético en el proceso de toma de decisiones asociadas a su rol.

2. Genera un ambiente facilitador del encuentro profesional con el profesor principiante.

3. Comunica ideas en los diversos escenarios de su actuación profesional como mentor.

4. Genera situaciones para la construcción de aprendizajes significativos por parte del profesor principiante.

5. Asesora al profesor principiante en el diseño, implementación y evaluación de propuestas pedagógicas pertinentes a los estudiantes y al contexto.

6. Asesora al profesor principiante en el proceso de autoevaluación de su propio desempeño profesional.

7. Distingue áreas y acciones de apoyo crítico para la autogestión del desarrollo profesional del profesor principiante.

8. Evalúa el impacto de los procesos de mediación profesional implementados.

9. Gestiona el tiempo en función de las necesidades.

10. Promueve que el profesor principiante se constituya en agente de cambio.

11. Genera redes para la inserción del profesor principiante con la realidad laboral y los contextos de desempeño. 
